\title{
Accretion funnels onto weakly magnetized young stars
}

\author{
N. Bessolaz ${ }^{1}$, C. Zanni ${ }^{1}$, J. Ferreira ${ }^{1}$, R. Keppens ${ }^{2,3,4}$, and J. Bouvier ${ }^{1}$ \\ 1 Laboratoire d'Astrophysique de Grenoble, Université Joseph Fourier, CNRS UMR5571, France \\ 2 Centre for Plasma Astrophysics, K.U. Leuven, Belgium \\ FOM Institute for Plasma Physics, Rijnhuizen, The Netherlands \\ 4 Astronomical Institute, Utrecht University, The Netherlands
}

Received 20 July 2007 / Accepted 11 November 2007

\section{ABSTRACT}

\begin{abstract}
Aims. We re-examine the conditions required to steadily deviate an accretion flow from a circumstellar disc into a magnetospheric funnel flow onto a slow rotating young forming star.

Methods. New analytical constraints on the formation of accretion funnels flows due to the presence of a dipolar stellar magnetic field disrupting the disc are derived. The Versatile Advection Code is used to confirm these constraints numerically. Axisymmetric MHD simulations are performed, where a stellar dipole field enters the resistive accretion disc, whose structure is self-consistently computed.

Results. The analytical criterion derived allows to predict a priori the position of the truncation radius from a non perturbative accretion disc model. Accretion funnels are found to be robust features which occur below the co-rotation radius, where the stellar poloidal magnetic pressure becomes both at equipartition with the disc thermal pressure and is comparable to the disc poloidal ram pressure. We confirm the results of Romanova et al. $(2002, \mathrm{ApJ}, 578,420)$ and find accretion funnels for stellar dipole fields as low as $140 \mathrm{G}$ in the low accretion rate limit of $10^{-9} M_{\odot} \mathrm{yr}^{-1}$. With our present numerical setup with no disc magnetic field, we found no evidence of winds, neither disc driven nor X-winds, and the star is only spun up by its interaction with the disc.

Conclusions. Weak dipole fields, similar in magnitude to those observed, lead to the development of accretion funnel flows in weakly accreting T Tauri stars. However, the higher accretion observed for most T Tauri stars $\left(\dot{M} \sim 10^{-8} M_{\odot} \mathrm{yr}^{-1}\right)$ requires either larger stellar field strength and/or different magnetic topologies to allow for magnetospheric accretion.
\end{abstract}

Key words. accretion, accretion disks - magnetohydrodynamics (MHD) - methods: numerical - stars: pre-main sequence

\section{Introduction}

Classical T-Tauri stars (CTTS) are magnetically active, show evidence for circumstellar accretion discs, and can have mean photospheric magnetic field magnitudes around $1 \mathrm{kG}$ (e.g. Johns-Krull et al. 1999). Such a strong stellar field is enough to disrupt the inner accretion disc, provided that one really measures the large scale magnetic field and not only local strong multipolar components from starspots. However, this does not seem to be the case since recent polarimetric measurements (Valenti \& Johns-Krull 2004) indicate a weak dipolar component lower than $200 \mathrm{G}$. Moreover, observations show evidence for non-direct accretion. Inverse P-Cygni profiles with strong redshift absorption wings are indicative of polar accretion near free-fall velocities along magnetospheric field lines from the inner disc edge (Edwards et al. 1993; Bouvier et al. 1999, 2003).

Although the magnetic field structure of these stars is probably complex (Gregory et al. 2006), dynamical models of stardisc interaction usually assume an aligned dipole field, to simplify the analytical work. Under this assumption, stellar field lines threading the Keplerian disc below the co-rotation radius $r_{\mathrm{co}}=\left(G M_{*} / \Omega_{*}^{2}\right)^{1 / 3}$, where $\Omega_{*}$ is the stellar angular velocity, would lead to a spin-up of the star, versus those beyond $r_{\mathrm{co}}$, to a spin-down. The radial extent of angular momentum exchange between the star and the disc is then determined by (1) the disc truncation radius $r_{\mathrm{t}}$, where the magnetic dipole diverts the radially accreting flow to funnel flows; and (2) an outer radius $r_{\text {out }}$ beyond which no more stellar field lines are connected to the disc. In this framework, a star-disc interaction occurring on a large radial extension (as proposed by Ghosh \& Lamb 1979; Cameron \& Campbell 1993; Armitage \& Clarke 1996), may lead to a disc-locking situation where the star remains at a slow rotation rate, despite accretion. On the other hand, it has been argued that this scenario is unlikely, since the stellar field lines would be opened up by differential rotation until severing this causal link (Aly \& Kuijpers 1990; Lovelace et al. 1995; Matt \& Pudritz 2005 , for a recent discussion on that issue). The outcome of this latter scenario would be a star-disc interaction limited to a small radial extension around the disc truncation radius. Many theoretical models then assume that the disc inner edge should be close to the co-rotation radius (Königl 1991; Shu et al. 1994) for the sake of angular momentum equilibrium.

For a decade, many numerical works have investigated the star-disc interaction issue (Hayashi et al. 1996; Kuker et al. 2003; Long et al. 2005). However, the formation of accretion curtains seems difficult to reproduce. Although Miller \& Stone (1997) found such polar accretion in the case of a $\mathrm{kG}$ dipolar stellar field associated with a disc field in the same direction to the latter, Romanova et al. (2002) were the first to demonstrate magnetospheric accretion along stellar field lines for $\mathrm{kG}$ pure dipolar field in axisymmetric simulations and next by performing 3D simulations (Romanova et al. 2003).

In this paper, we address the issue of the disc truncation radius and its localisation as a function of the disc (accretion rate) and stellar (dipole field) parameters. In Sect. 2 we provide analytical constraints for driving steady accretion funnels and derive an estimate of the position of the truncation radius. We then use 
numerical MHD simulations in Sect. 3 to verify this prediction. We confirm magnetospheric accretion for a slowly rotating star with an inner disc hole and a weak stellar magnetic field compatible with observations of weak accretors.

\section{The disc truncation radius}

For a given accretion disc model, predicting where the truncation by the stellar magnetosphere will occur is an important issue. Different estimates were given in the literature. We consider here a pure dipolar field with a strength at the stellar surface in the equatorial plane equal to $B_{*}$.

A first dimensional estimate expresses the truncation radius $r_{\mathrm{t}}$ in the form $r_{\mathrm{t}}=k r_{\mathrm{A}}$ where the Alfvén radius

$r_{\mathrm{A}}=\left(\frac{B_{*}^{4} R_{*}^{12}}{2 G M_{*} \dot{M}_{a}^{2}}\right)^{1 / 7}$

is a characteristic length which can be derived by equating the ram pressure of a free-falling spherical envelope with the magnetic pressure of a dipolar field (Elsner \& Lamb 1977). Different estimates of the adimensional coefficient $k$ have been given in the literature, ranging from 0.5 (Ghosh \& Lamb 1979; Königl 1991; Long et al. 2005) to 1 (Arons 1993; Ostriker \& Shu 1995; Wang 1996).

A second criterion (Cameron \& Campbell 1993; Armitage \& Clarke 1996; Matt \& Pudritz 2005, and references therein) states that accretion funnels will take place when the magnetic torque due to the stellar field matches the "viscous" or turbulent torque, namely

$-J_{r} B_{z} \simeq \frac{B_{\phi}^{+} B_{z}}{\mu_{\mathrm{o}} h}=\frac{1}{r^{2}} \frac{\partial}{\partial r} \eta_{v} r^{3} \frac{\partial \Omega}{\partial r} \simeq-\alpha_{v} \frac{P}{r}$

where $B_{\phi}^{+}$is the toroidal field at the disc surface, $h$ the disc scale height, $P$ the disc pressure and $\alpha_{v}$ the Shakura \& Syunyaev (1973) parameter. This criterion (hereafter A) gives an upper limit for the truncation radius, as it only defines a radius where the star-disc interaction starts to affect accretion. This maximal truncation radius $r_{\mathrm{t} \text {, max }}$ occurs when the plasma beta, defined as $\beta=2 \mu_{\mathrm{o}} \mathrm{P} / \mathrm{B}_{\mathrm{z}}^{2}$, becomes $\beta=2 q /\left(\alpha_{v} \varepsilon\right)$ where $q=\left|B_{\phi}^{+} / B_{z}\right|$ is a measure of the magnetic shear and $\varepsilon=h / r$ is the disc aspect ratio. In a thin Keplerian accretion disc, one gets $\beta \gg 1$ at $r_{\mathrm{t}, \max }$ since $q$ is close to unity to avoid opening of the magnetosphere (e.g. Matt \& Pudritz 2005).

A third criterion represents a more conservative approach,

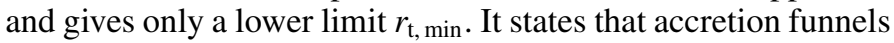
take place when accretion is no longer possible because of the overwhelming field strength. It is usually written (Koldoba et al. 2002; Romanova et al. 2002)

$\frac{B^{2}}{\mu_{\mathrm{o}}}=\rho v^{2}+P$

where $v$ is the total speed. Note that this criterion is not predictive since we cannot calculate a priori the dominant azimuthal velocity $v_{\phi}$ as it is itself an outcome of the star-disc interaction. Nevertheless, if one wishes to provide an estimate of the truncation radius, then we can use instead $B_{z}^{2} / \mu_{o}=\rho v_{\phi}^{2}$, where $v_{\phi}=v_{K}=\sqrt{G M_{*} / r}$ and a negligible thermal pressure (both approximations are valid in a thin disc). This will be our criterion $B$. At the truncation radius estimated as such, the plasma beta is $\beta=2 \varepsilon^{2} \ll 1$.
From the previous discussion, it appears quite obvious that $\beta \sim 1$ is a better approximation for $r_{\mathrm{t}}$. The stellar magnetic field, which is bound to become dominant in the magnetosphere, must first favour accretion, i.e. the magnetic torque must be negative. If this is not the case, namely if $r>r_{t, \max }>r_{\mathrm{co}}$, the disc material is radially expelled. This is the "propeller" regime as studied e.g. by Ustyugova et al. (2006) and references therein. Accretion thus implies $r_{\mathrm{t}}<r_{\mathrm{co}}$, with stellar magnetic field lines as leading spirals. Below co-rotation, accretion will proceed quite naturally thanks to both the viscous and the stellar torques. There are then two more independent constraints that must be fulfilled to produce steady funnel flows. First, the accretion flow must be prevented by the presence of the magnetosphere. The simplest way to express this is to require that the magnetic poloidal pressure balances the accretion ram pressure $\rho u_{r}^{2}$. This defines a radius $r_{\mathrm{bf}}$ where

$\beta \simeq m_{\mathrm{s}}^{-2}$

where $m_{\mathrm{s}}=u_{r} / C_{\mathrm{s}}$ is the sonic Mach number measured at the disc midplane. Now, at radii $r<r_{t, \max }$, accretion is mainly due to the stellar torque and $m_{\mathrm{s}}=4 q / \beta \gg \alpha \varepsilon$ : material is accreting to the star much faster than in the outer accretion disc. Second, material at the disc midplane must be lifted and loaded onto the stellar field lines. With a dipole field configuration, such a vertical motion can only be due to a vertical thermal plasma pressure gradient. It therefore requires that the magnetic field compression is not too strong. This leads naturally to an equipartition, $\beta \sim 1$ (as already proposed by Pringle \& Rees 1972; Aly 1980).

One important point to note is that once a large scale magnetic field is close to equipartition in an accretion disc, it is able to deviate a large fraction of the disc plasma from its radial motion to a vertical one. This has been shown with the calculations of magnetized accretion-ejection structures by Ferreira \& Pelletier (1995) and confirmed by numerical MHD simulations (Casse \& Keppens 2002; Zanni et al. 2007). In one sense, making funnel flows involves the same physics as loading mass in magnetized jets. In fact, almost all of the disc mass can be lifted and loaded onto the field lines when $\beta \sim 1$, depending mostly on the field bending (see Fig. 3 in Ferreira \& Pelletier 1995). This is why we assume, for finding a simple analytical criterion, that the disc truncation radius $r_{\mathrm{t}}$ is close to the radius where $\beta \sim 1$, namely $r_{\mathrm{t}} \sim r_{\mathrm{bf}}$. Using the above estimates, we derive the following two constraints:

$\beta \sim 1$ and $m_{\mathrm{s}} \sim 1$.

that must be fulfilled in order to provide steady state funnel flows. For a dipole field, this translates into a theoretical truncation radius

$\frac{r_{\mathrm{t}, \mathrm{th}}}{R_{*}} \simeq 2 m_{\mathrm{s}}^{2 / 7} B_{*}^{4 / 7} \dot{M}_{a}^{-2 / 7} M_{*}^{-1 / 7} R_{*}^{5 / 7}$

where the stellar field $B_{*}$ has been normalised to $140 \mathrm{G}$, disc accretion rate $\dot{M}_{a}$ to $10^{-8} M_{\odot} \mathrm{yr}^{-1}$, stellar mass to $0.8 M_{\odot}$ and stellar radius to $2 R_{\odot}$. Note that these are all typical values for CTTS while the chosen value of the magnetic stellar field $B_{*}$ is consistent with observations of dipole fields in such objects (see Valenti \& Johns-Krull 2004; Bouvier et al. 2007, and references therein). It is clear that the conditions given by Eq. (5) state that the accretion speed close to the base of the accretion funnel is of the order of the Alfvén speed. Indeed Eq. (6) can be rewritten as $r_{\mathrm{t}, \text { th }} \sim m_{\mathrm{s}}^{2 / 7} r_{\mathrm{A}}$, where the Alfvén radius for spherical accretion $r_{\mathrm{A}}$ was defined in Eq. (1). On the other hand, the co-rotation radius 
is $r_{\mathrm{co}} / R_{*}=7.8 M_{*}^{1 / 3} R_{*}^{-1} P_{*}^{2 / 3}$ for a typical 8-day stellar period $P_{*}$, which provides

$\frac{r_{\mathrm{t}, \text { th }}}{r_{\mathrm{co}}} \simeq 0.25 m_{\mathrm{s}}^{2 / 7} B_{*}^{4 / 7} \dot{M}_{a}^{-2 / 7} M_{*}^{-10 / 21} R_{*}^{12 / 7} P_{*}^{-2 / 3}$.

Remarkably, taking typical values for CTTS with a low magnetic dipole field gives a theoretical truncation radius not only smaller than the co-rotation radius but also consistent with observations of inner disc holes (Najita et al. 2003). This is in strong contrast with unobserved large scale $\mathrm{kG}$ fields usually used in the literature. This implies that the formation of funnel flows should always spin up the star unless (i) enough stellar field lines remain connected to the disc beyond $r_{\mathrm{co}}$ and/or (ii) ejection of stellar angular momentum is indeed taking place. In the following section, 2.5D numerical MHD simulations are used to show that the conditions in (5) are indeed those prevailing at the disc inner edge. If this is true, then we should recover a truncation radius located at our theoretical estimate (6) for a low dipole field of $140 \mathrm{G}$.

\section{Numerical experiments}

\subsection{Equations and numerical setup}

We use the $\operatorname{VAC}^{1}$ code (Tóth 1996) to solve the full set of axisymmetric dimensionless resistive MHD equations (with the magnetic permeability $\left.\mu_{0}=1\right)$ in cylindrical coordinates $(r, z)$ :

$\frac{\partial \rho}{\partial t}+\nabla \cdot\left(\rho \boldsymbol{v}_{\mathrm{p}}\right)=0$

$\frac{\partial \rho \boldsymbol{v}}{\partial t}+\nabla \cdot(\rho \boldsymbol{v} \boldsymbol{v}-\boldsymbol{B B})+\nabla\left(\frac{B^{2}}{2}+P\right)=-\rho \nabla \Phi_{G}$,

$\frac{\partial \boldsymbol{B}}{\partial t}+\nabla \cdot(\boldsymbol{v} \boldsymbol{B}-\boldsymbol{B} \boldsymbol{v})=\nabla \times[\eta(\nabla \times \boldsymbol{B})]$,

$\frac{\partial E}{\partial t}+\nabla \cdot\left[\left(E+\frac{B^{2}}{2}+P\right) \boldsymbol{v}-\boldsymbol{B}(\boldsymbol{B} \cdot \boldsymbol{v})\right]=\eta J^{2}-\boldsymbol{B} \cdot(\nabla \times \eta \boldsymbol{J})$,

where $\rho$ is the plasma density, $\boldsymbol{v}_{\mathrm{p}}$ the poloïdal velocity, $\boldsymbol{v}$ the total velocity, $\boldsymbol{B}$ the magnetic field, $P$ the thermal pressure, $\eta$ the magnetic resistivity, $\boldsymbol{J}=\nabla \times \boldsymbol{B}$ the current density, $\Phi_{G}=$ $-\frac{G M_{*}}{\left(r^{2}+z^{2}\right)^{1 / 2}}$ the gravity potential created by the central star and $E=\frac{P}{\gamma-1}+\rho \frac{v^{2}}{2}+\frac{B^{2}}{2}+\rho \Phi_{G}$ the total energy density. The gravity is treated as a source term in the momentum and energy equations of VAC.

Time evolution is done with a conservative second order accurate total variation diminishing Lax Friedrichs scheme with minmod limiters applied on primitive variables, except for density where a van Leer limiter is used instead to better resolve contact discontinuities. Powell source terms are used to ensure the divergence free property of the magnetic field and the code has been modified so as to compute only the deviations from the dipolar component (Tanaka 1994; Powell et al. 1999). The splitting technique has three main advantages. First, it is crucial to properly represent an initial force-free configuration numerically. Second, since the total conserved energy contains only the energy associated with the deviation from the dipolar field, this method improves the computation of the thermal energy in low $\beta$ regions. Third, when used in association with the Powell method, it helps control the divergence of the magnetic field, since only the deviation from the background field is used to

\footnotetext{
${ }^{1}$ http://www.phys.uu.nl/ toth/
}

calculate the divergence and the Powell source terms. The divergence method used here gives $|\nabla \cdot \boldsymbol{B}| \Delta R \leq 0.01|\boldsymbol{B}|$ but simulations done with PLUTO $^{2}$ and a constraint-transport scheme show that our results are not strongly modified by this method (Zanni et al., in prep).

\subsection{Boundary conditions}

Boundary conditions take symmetric and asymmetric conditions for the axis and the disc midplane, while continuous (outflow) conditions are used at the outer edge. At the inner edge corresponding to the stellar surface, a linear extrapolation of density and pressure is made. After using free extrapolation for the poloidal velocity, this latter is forced to be parallel to the total poloidal magnetic field. The poloidal field is fixed, contrary to Romanova et al. (2002). We use a boundary condition on the toroidal magnetic field designed by Zanni et al. (in prep.). It allows us to derive $B_{\phi}$ by forcing the magnetic surfaces to locally rotate at the stellar velocity. In practice, the radial derivative of the toroidal field is computed from the angular momentum conservation equation where the temporal derivative giving the local acceleration is replaced by

$\rho \frac{\partial}{\partial t}(\Omega r)=\rho \frac{\left(\Omega_{*} r+v_{\mathrm{p}} B_{\phi} / B_{\mathrm{p}}-\Omega r\right)}{\Delta t}$

where the timescale $\Delta t=\Delta R / V_{\mathrm{A}}$ is the Alfvén crossing time of one cell $\Delta R$ at the inner edge of the simulation box. This allows the system to evolve without imposing any arbitrary conditions on $B_{\phi}$, and hence on the torques.

\subsection{Initial condition}

In the initial conditions we take a Keplerian disc surrounded by an adiabatic corona in hydrostatic (non rotating) equilibrium. The disc is adiabatic with an index $\gamma=5 / 3$ and an aspect ratio $\epsilon=h / r=C_{\mathrm{s}} / V_{K}=0.1$. The surface of the disc is determined by the pressure equilibrium between the disc and the corona, while the initial truncation radius has been chosen arbitrarily. The density and pressure expressions for both the disc and the corona are

$$
\begin{aligned}
& \rho_{\mathrm{d}}=\rho_{\mathrm{d} 0}\left[\frac{2}{5 \epsilon^{2}}\left(\frac{R_{0}}{\sqrt{r^{2}+z^{2}}}-\left(1-\frac{5}{2} \epsilon^{2}\right) \frac{R_{0}}{r}\right)\right]^{\frac{1}{\gamma-1}} \\
& P_{\mathrm{d}}=\epsilon^{2} V_{K 0}^{2} \rho_{\mathrm{d} 0}^{1-\gamma} \rho_{\mathrm{d}}^{\gamma} \\
& \rho_{\mathrm{c}}=\rho_{c 0}\left(\frac{R_{0}}{\sqrt{r^{2}+z^{2}}}\right)^{\frac{1}{\gamma-1}} \\
& P_{\mathrm{c}}=\frac{2}{5} V_{K 0}^{2} \rho_{\mathrm{c} 0}^{1-\gamma} \rho_{\mathrm{c}}^{\gamma}
\end{aligned}
$$

The density contrast between the disc and the corona is $\rho_{\mathrm{c} 0} / \rho_{\mathrm{d} 0}=$ 0.01 . A pure dipolar magnetic field is set up in the computational domain in equipartition with the thermal pressure of the disc at $r=R_{0}$. The rotation period of the star is set in order to place the corotation radius at $r=2 R_{0}$. The disc has no viscosity but is resistive with an alpha-like magnetic resistivity decreasing on a disc scale height, namely $\nu_{\mathrm{m}}=\alpha_{\mathrm{m}} \Omega_{\mathrm{k}} h^{2} \exp \left(-\left(\frac{z}{h}\right)^{4}\right)$. In this paper, we fix $\alpha_{\mathrm{m}}=0.1$. The initial poloidal flow within the disc is zero and the disc is slightly sub-Keplerian with $v_{\phi}=\sqrt{1-\frac{5}{2} \epsilon^{2}} \sqrt{\frac{G M_{*}}{r}}$.

\footnotetext{
2 A description of this code can be found in Mignone et al. (2007).
} 

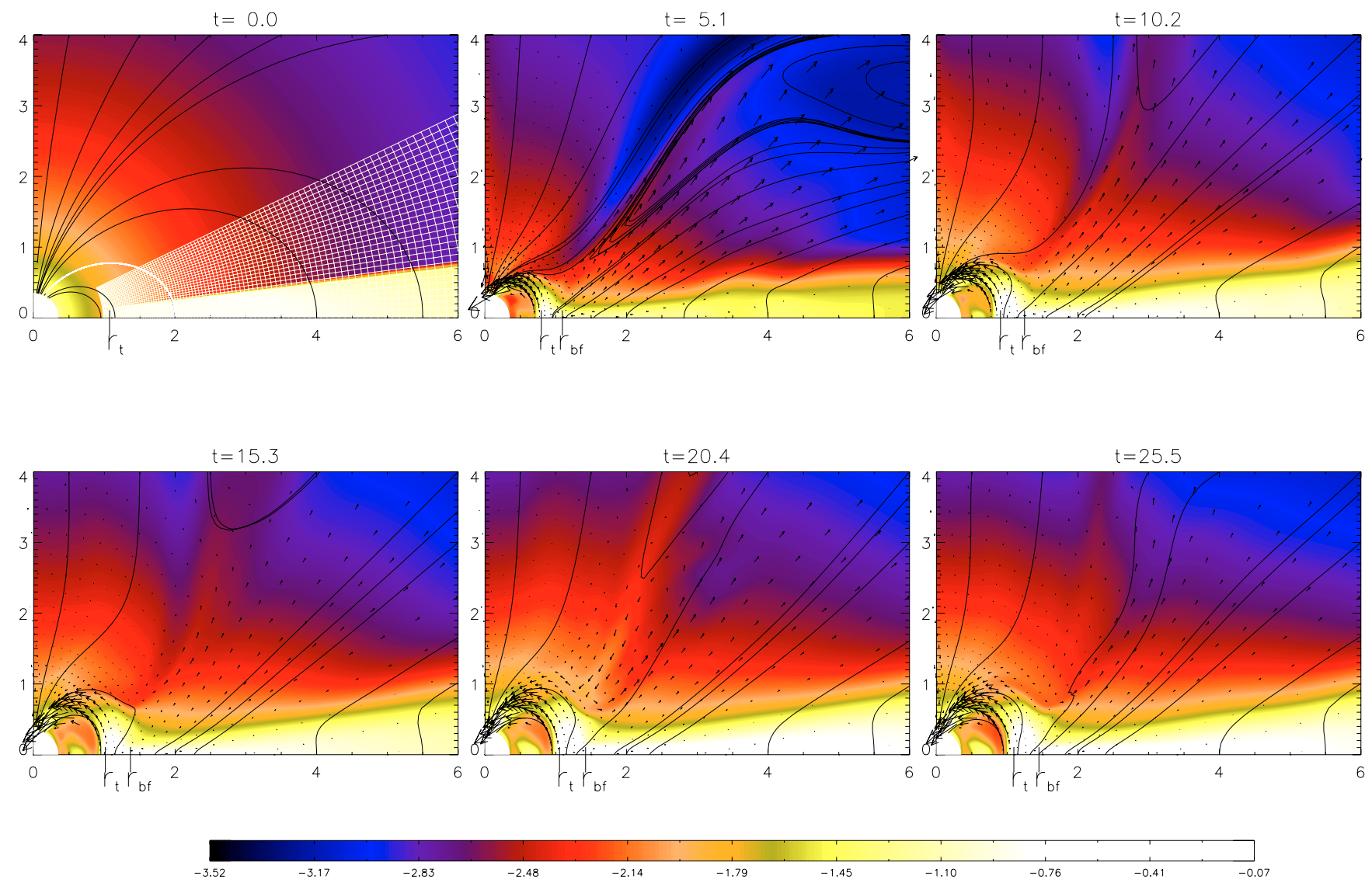

Fig. 1. Resistive MHD simulation for a 5-day period CTTS with $B_{*}=141 \mathrm{G}$ and $\alpha_{\mathrm{m}}=0.1$ after $t=0,5.1,10.2,15.3,20.4,25.5$ Keplerian periods at the disc inner edge corresponding to a physical time of 1.5 months. We show the density distribution in the computational domain using a log scale. The black lines draw the magnetic field lines and the black arrows represent the velocity field. The white line on the first snapshot represents an initial magnetic field line anchored at $r_{\mathrm{co}}$. We superimpose a part of the computational grid to show the good resolution near the truncation radius. An accretion column is formed between $r_{\mathrm{t}}$ and $r_{\mathrm{bf}}$ (see text) and one observes the expansion of the poloidal magnetic field and transient disc ejecta. The accretion rate at the stellar surface is equal to $1.9 \times 10^{-9} M_{\odot} \mathrm{yr}^{-1}$ at $\mathrm{t}=5$ and stabilises around $0.91 \times 10^{-9} M_{\odot} \mathrm{yr}^{-1}$ at $t=15$. No $\mathrm{X}$-winds are formed and the star is being spun up.

The grid and stellar rotation period were chosen to allow good resolution at the truncation radius $R_{0}$ while maintaining the co-rotation radius at $r=2 R_{0}$ well inside the domain. Our polar grid of $N_{R} \times N_{\theta}=170 \times 100$ is stretched in the spherical $R$ direction (see Fig. 1) and goes from $R_{\min }=0.35 R_{0}$ at the stellar surface to $R_{\max }=10.35 R_{0}$.

The results are presented in adimensional units: lengths are given in units of $R_{0}$, which corresponds to the truncation radius of the reference simulation (see Sect. 3.5); speeds are expressed in units of the Keplerian speed $V_{K 0}=\sqrt{G M_{*} / R_{0}}$ and densities in units of $\rho_{\mathrm{d} 0}$, which is the initial disc density at $\left(r=R_{0}, z=0\right)$. Time is given in units of the Keplerian period at $R_{0}$, i.e. $t_{0}=2 \pi R_{0} / V_{K 0}$. Mass accretion rates is given in units of $\dot{M}_{0}=\rho_{0} V_{K 0} R_{0}^{2}$ while we express the torques in units of $\dot{L}_{0}=\rho_{0} V_{K 0}^{2} R_{0}^{3}$.

\subsection{Reference values}

We consider a $M_{*}=0.8 M_{\odot}$ young star with a radius of $R_{*}=2 R_{\odot}$ and a pure stellar dipole field with $B_{*}=141 \mathrm{G}$. With these assumptions the normalisation units will be $R_{0}=2.86 R_{*}=$ $4 \times 10^{9} \mathrm{~m}, V_{K 0}=\sqrt{G M_{*} / R_{0}} \simeq 1.63 \times 10^{5} \mathrm{~m} \mathrm{~s}^{-1}$ and $\rho_{d 0}=$ $\left(B_{*}^{2} R_{*}^{6}\right) /\left(2 \mu_{0} \epsilon^{2} V_{K 0}^{2} R_{0}^{6}\right) \simeq 5.51 \times 10^{-10} \mathrm{~kg} \mathrm{~m}^{-3}$. Since we place the corotation radius at $2 R_{0}$, the rotation period of the star is $P_{*}=2 \pi \sqrt{8 R_{0}^{3} / G M_{*}} \simeq 5.1$ days while the time will be scaled in units of $t_{0}=2 \pi R_{0} / V_{K 0}=1.54 \times 10^{5} \mathrm{~s} \simeq 1.78$ days. The computational domain extends up to $0.3 \mathrm{AU}$. Finally the normalisation for the accretion rates is given by $\dot{M}_{0}=\rho_{0} V_{K 0} R_{0}^{2} \simeq$ $2.27 \times 10^{-8} M_{\odot} \mathrm{yr}^{-1}$.

From these reference values, simulations done here can be scaled for another range of parameters $\left(R_{*}, M_{*}, B_{*}\right)$ in the following way:

$$
\begin{aligned}
R_{0} & =4 \times 10^{9}\left(\frac{R_{*}}{2 R_{\odot}}\right) \mathrm{m} \\
V_{K 0} & =1.63 \times 10^{5}\left(\frac{M_{*}}{0.8 M_{\odot}}\right)^{1 / 2}\left(\frac{R_{*}}{2 R_{\odot}}\right)^{-1 / 2} \mathrm{~m} \mathrm{~s}^{-1} \\
\rho_{0} & =5.51 \times 10^{-10}\left(\frac{R_{*}}{2 R_{\odot}}\right)\left(\frac{B_{*}}{141 \mathrm{G}}\right)^{2}\left(\frac{M_{*}}{0.8 M_{\odot}}\right)^{-1} \mathrm{~kg} \mathrm{~m}^{-3} \\
P_{*} & =5.1\left(\frac{R_{*}}{2 R_{\odot}}\right)^{3 / 2}\left(\frac{M_{*}}{0.8 M_{\odot}}\right)^{-1 / 2} \text { days, } \\
t_{0} & =1.54 \times 10^{5}\left(\frac{R_{*}}{2 R_{\odot}}\right)^{3 / 2}\left(\frac{M_{*}}{0.8 M_{\odot}}\right)^{-1 / 2} \mathrm{~s} \\
\dot{M}_{0} & =2.27 \times 10^{-8}\left(\frac{R_{*}}{2 R_{\odot}}\right)^{5 / 2}\left(\frac{B_{*}}{141 \mathrm{G}}\right)^{2}\left(\frac{M_{*}}{0.8 M_{\odot}}\right)^{-1 / 2} M_{\odot} \mathrm{yr}^{-1}
\end{aligned}
$$




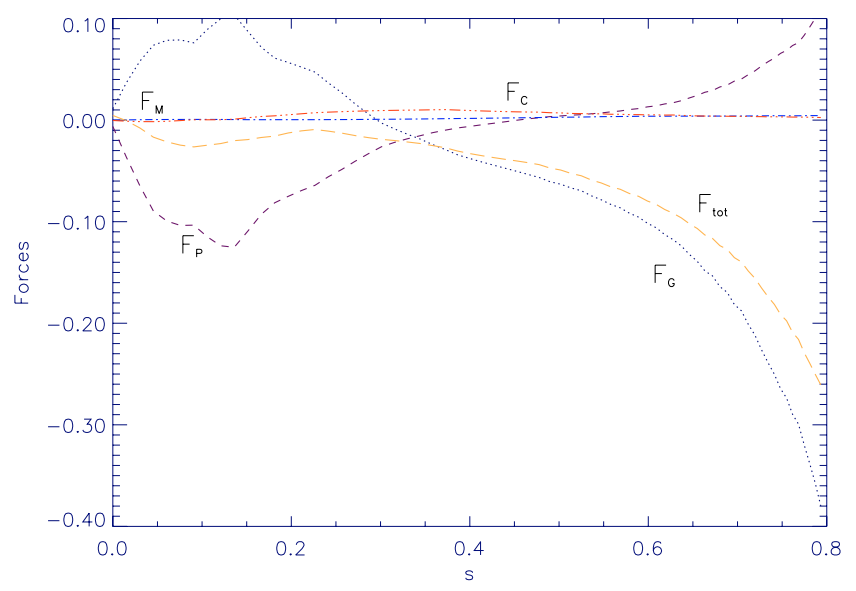

Fig. 2. Projection of the forces in normalised units along a magnetic field line in the middle of the accretion column, for run (s1) at $t=$ 10 . We represent the gravity $F_{\mathrm{G}}$, the centrifugal force $F_{\mathrm{C}}$, the thermal pressure gradient $F_{\mathrm{P}}$, the poloidal magnetic force $F_{\mathrm{M}}$ and the total force $F_{\text {tot }}$ as a function of the curvilinear coordinate s. The disc midplane is located at $s=0$ and the disc surface corresponds to $s \sim 0.2$. Gravity begins to dominate the dynamics only at some distance between the disc and the $\operatorname{star}(s \sim 1.2$ at the stellar surface $)$.

\subsection{Methodology}

For these fixed stellar and accretion disc parameters, we make three simulations with different initial truncation radius $r_{\text {to }}$. Our reference simulation (s1) corresponds to an initial truncation radius fulfilling Eq. (6), namely $r_{\text {to }}=1$ with $\beta\left(r_{\text {to }}\right)=1$. Simulation (s2) is done for $r_{\text {to }}=1.5$ with $\beta\left(r_{\text {to }}\right)=10$ and simulation (s3) for $r_{\text {to }}=0.5$ with $\beta\left(r_{\text {to }}\right)=0.1$.

We then let the system evolve and observe whether or not the real disc truncation radius $r_{\mathrm{t}}$ converges towards the theoretical radius $r_{\mathrm{t}, \text { th }}$ as given by Eq. (6). With our normalised quantities, the truncation radii in simulations $(\mathrm{s} 2)$ and $(\mathrm{s} 3)$ are thus expected to converge towards 1 , with $\beta\left(r_{\mathrm{t}}\right) \sim 1$. To check this, we identify this radius and then compute the plasma beta. It is however not straightforward to define this radius since the magnetic field is not a solid wall: the radial to vertical deviation of the flow is quite smooth. In practice, we get the truncation radius $r_{\mathrm{t}}$ by detecting a steep decrease in density at the disc midplane (see Fig. 4).

\subsection{Results}

Figure 1 shows a series of snapshots of our reference simulation (s1). The upper left panel shows the initial condition. We have superimposed a part of the computational grid to show the resolution achieved. We have a resolution of 8 points in the vertical direction within the disc at each radius, while the resolution within the accretion column reaches 20 points at the stellar surface. Magnetic field lines are in black while the white line traces a magnetic field line connecting the star to the corotation radius $r_{\mathrm{co}}=2$. After a rapid transient phase with the opening of stellar field lines, a quasi-steady situation is achieved where quasi-steady accretion columns are formed, even with a low stellar dipole field. The final (equilibrium) truncation radius is $r_{\mathrm{t}} \sim 1.1-1.2$, and thus well below the co-rotation radius.

Figure 2 shows the projection $F=\boldsymbol{B}_{\mathrm{p}} \cdot \boldsymbol{F} /\left|\boldsymbol{B}_{\mathrm{p}}\right|$ of the various forces along a magnetic field line located at the centre of the accretion column at $t=10$. Since the stellar magnetic moment is directed northwards, a negative force is actually pulling material upwards. Accretion is achieved because of the negative magnetic torque (not shown here) but against the poloidal magnetic force
$F_{\mathrm{M}}$ which tends to prevent it. Note however that it is negligible with respect to the other forces. What drives the poloidal motion in the accretion column is actually the plasma pressure gradient $F_{\mathrm{P}}$ which was built in by the accumulation of accreting mass and allows it to be lifted up and loaded onto closed stellar field lines. It acts exactly as in accretion-ejection structures, enabling the necessary transition from the resistive MHD disc to the ideal MHD columns (Ferreira \& Pelletier 1995). The plasma pressure gradient remains dominant well above the disc surface, located around the curvilinear coordinate $s=0.2$. Given the dipole topology, this is not surprising as material must first be lifted against gravity ( $F_{\mathrm{G}}$ is initially positive) by $F_{\mathrm{P}}$. Then, at some point (which depends mostly on the dipole geometry) gravity overcomes it and becomes the leading agent. Again, this is only possible because the centrifugal term $F_{\mathrm{C}}$ plays almost no role due to the azimuthal magnetic braking. Matter then reaches the star in a dynamical time scale with approximately free-fall velocities $\left(v_{\text {pol }} \approx 310 \mathrm{kms}^{-1}\right)$.

The strong differential rotation beyond $r_{\mathrm{t}}$, in a region where $\beta \gg 1$, leads to an expansion of the poloidal magnetic field lines. Such an expansion starts at the inner regions and enforces the outer field lines to inflate as well (but the cause there is not the differential rotation). Once these loops (inflated lines) reach the outer boundary of the computational domain, they open, mimicking a reconnection. This opening is actually an effect of the boundary conditions used but, for all practical means, we see no strong bias on the evolution of the system. Anyway, on quite short time scales $(t \sim 3)$, most of the stellar magnetic flux not related to the accretion funnels around $r_{\mathrm{t}}$ has been opened (Fig. 1). One might think that a disc wind is driven in this region of the disc as the Blandford \& Payne (1982) criterion is fulfilled. Moreover, some mass is indeed leaving the disc along these open field lines. But this is only a breeze and not a proper jet: the ejected material does not reach super-Alfvénic speeds. We note also that no X-winds (Shu et al. 1994) are obtained despite the favourable magnetic configuration ${ }^{3}$. The reason why no disc wind is obtained, neither extended nor X-wind, is that the field threading the disc in these regions is far below equipartition.

The results of varying the initial truncation radius (simulations s2 and s3) are summarised in Fig. 3. In both cases, we observe a rapid convergence to $r_{\mathrm{t}} \approx 1.1-1.2$ on a dynamical time scale. Although some fluctuations in time can be seen in $r_{\mathrm{t}}$, it remains strikingly close to unity. Besides, it is really close to the radius of equipartition where $\beta=1, r_{1}$, with a relative position lower than $4 \%$. This is another indication of the importance of the plasma pressure gradient in defining the truncation radius and justifies the approximation $\beta\left(r_{\mathrm{t}}\right) \sim 1$.

The accretion rate onto the star is that which is actually observationally determined through, e.g. veiling measurements. It is obtained here by computing the mass flux in the accretion column, namely $\dot{M}_{a}=\int_{S} \rho \boldsymbol{v}_{\mathrm{p}} \cdot \mathrm{d} \boldsymbol{S}=-4 \pi R_{*}^{2} \int_{S} \rho v_{R} \sin \theta \mathrm{d} \theta$. Surprisingly, it is found to converge towards $0.91 \times 10^{-9} M_{\odot} \mathrm{yr}^{-1}$ (see Fig. 5), hence a factor 10 smaller than the mean accretion rate in CTTS. We will come back to this issue later. If we now insert this value into Eq. (6), we find a theoretical truncation radius $r_{\mathrm{t}, \mathrm{th}}=1.38$, using $m_{\mathrm{s}}=1$. This is off by $20 \%$, which is not bad considering our crude approximations of such a complicated problem.

${ }^{3}$ This is a direct simulation of the scenario invoked by these authors since the disc magnetic field comes from the opening of the magnetosphere. 


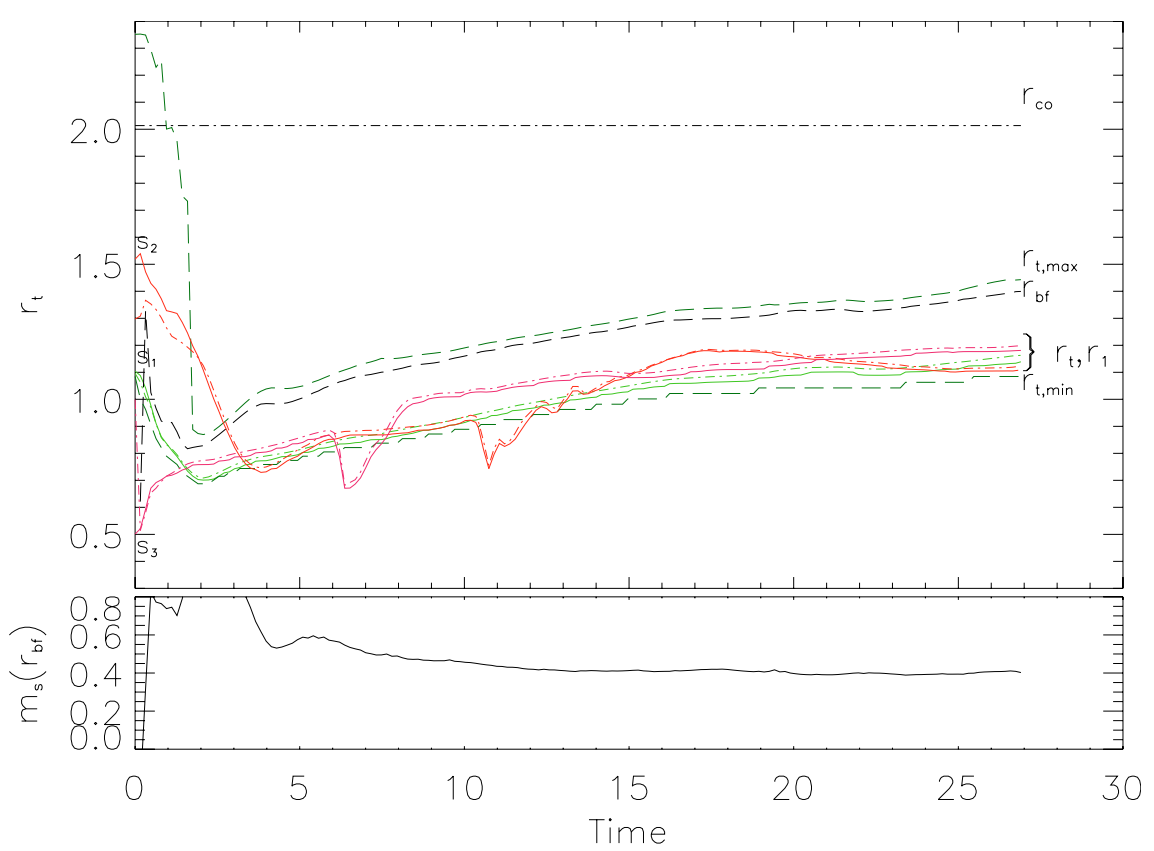

Fig. 3. Top: evolution in time of the position of the truncation radius $r_{\mathrm{t}}$ (solid lines) for a set of resistive MHD simulations with $B_{*}=141 \mathrm{G}$, $P_{*}=5.1$ days and $\alpha_{\mathrm{m}}=0.1$ and a different initial truncation radius. The time unit is the Keplerian period at $r=1$. All runs converge towards a truncation radius $r_{\mathrm{t}} \sim 1.2$ (solid lines). Dash-dot lines represent, for each simulation, the radius $r_{1}$ where $\beta=1$. We report also the radius $r_{\mathrm{bf}}$ (dashed line) which indicates the base of the funnel flow, $r_{\mathrm{t}, \max }$ (dashed line) and $r_{\mathrm{t}, \min }$ for our reference run (s1). Bottom: evolution in time of the sonic Mach number at the base of the funnel, $m_{\mathrm{s}}\left(r_{\mathrm{bf}}\right)$, for run $\left(s_{1}\right)$.

Since $\beta\left(r_{\mathrm{t}}\right) \sim 1$ is well verified, the main source of discrepancy in Eq. (6) is due to the assumption of $m_{\mathrm{s}}\left(r_{\mathrm{t}}\right) \sim 1$. This is too crude for an obvious reason. Indeed, the disc truncation radius, as measured by the steep drop in density at the equatorial plane, is actually the point where $u_{\mathrm{r}}=0$, hence $m_{\mathrm{s}}=0$. Figure 3 shows the evolution of the sonic Mach number $m_{\mathrm{s}}$ computed for run (s1) at $r_{\mathrm{bf}}$, which corresponds to the base of the funnel flow where the poloidal magnetic pressure matches the poloidal ram pressure. It can be seen that $r_{\mathrm{bf}}$ is larger than $r_{\mathrm{t}}$ by almost $30 \%$. This is not surprising as it is necessary to first brake efficiently material $\left(r_{\mathrm{bf}}\right)$ before being able to lift it up $\left(r_{\mathrm{t}}\right)$. This is also illustrated in Fig. 4 where we plot the radial profile of several quantities at the disc midplane: density $\rho$, angular velocities (real $\Omega$ and Keplerian $\Omega_{\mathrm{K}}$ ) and $m_{\mathrm{s}}$. Clearly, taking $m_{\mathrm{s}}=1$ to derive $r_{\mathrm{t}, \text { th }}$ is too crude. We find that using a value of $m_{\mathrm{s}} \simeq 0.45$, namely close to the real value $m_{\mathrm{s}}\left(r_{\mathrm{bf}}\right)$ (see Fig. 3), provides a much better estimate with $r_{\mathrm{r}, \text { th }}$ close to $r_{\mathrm{t}}$ with an accuracy better than $10 \%$.

Although the simulations here do not take into account viscosity, for completeness, we plotted in Fig. 3 the evolution of the radius $r_{\mathrm{t}, \max }$ for run (s1). To do so, we assumed $\alpha_{v}=1$ (thus overestimating the viscous torque) and measured numerically $q$ (see Sect. 2). As expected, $r_{\mathrm{t}, \text { max }}$ remains always significantly larger than the real truncation radius $r_{\mathrm{t}}$, which implies that criterion $\mathrm{A}$ is not good enough. On the other hand, criterion $B$ would give a truncation radius located at $r_{\mathrm{t}}=0.3$ : no accretion column should have been observed at all in our simulations. Our results clearly show that criterion $B$ is not relevant. What about the criterion as expressed in Eq. (3)? We plotted in Fig. 3 the evolution of the radius $r_{\mathrm{t}, \min }$ given by this equation and computed for run (s1). It turns out that it gives a good (though under-) estimate of the real truncation radius $r_{\mathrm{t}}$ as already pointed out by Romanova et al. (2002). This is because the star disc interaction introduces a sharp decrease in both the disc midplane density $\rho$ and azimuthal velocity $v_{\phi}=\Omega r$ (see Fig. 4). However, as stressed in

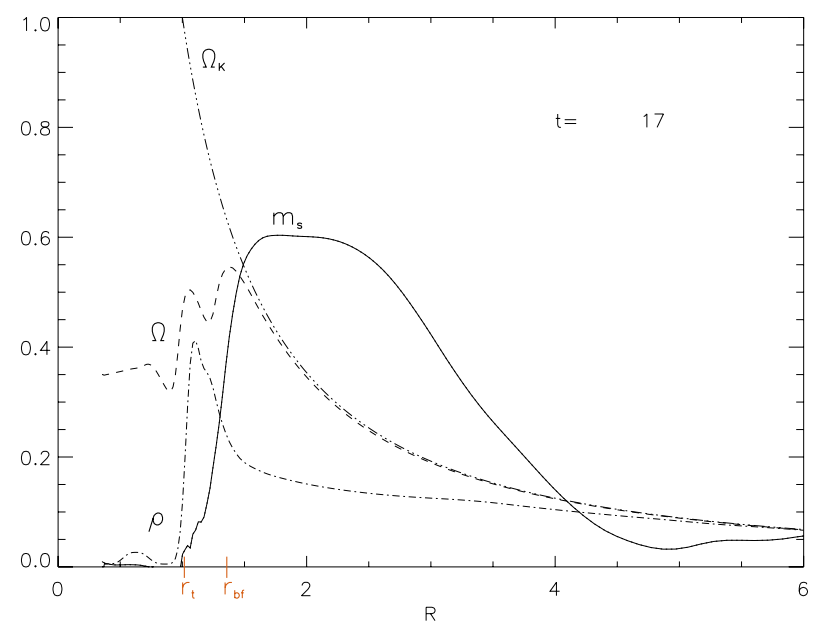

Fig. 4. Radial distributions of density $\rho$, angular velocities $\Omega$ (real) and $\Omega_{\mathrm{K}}$ (Keplerian) and sonic Mach number $m_{\mathrm{s}}=u_{r} / C_{\mathrm{s}}$ at the disc midplane, for run (s1) after 17 Keplerian rotations. Notice the large accretion velocity in the region of opened stellar field lines.

Sect. 2, while basically correct, such a criterion is meaningless as a predictive tool.

As far as the formation of funnel flows is concerned, we fully confirm the results of Romanova et al. (2002): these flows are indeed robust features of axisymmetric MHD simulations. Their different boundary conditions on the magnetic field at the stellar surface, namely a fixed normal component and free conditions for $B_{\theta}$ and $B_{\phi}$, do not finally play any significant role for the truncation of the disc and the formation of accretion columns. We have also done simulations with other values of the magnetic resistivity parameter $\alpha_{\mathrm{m}}$ with no change in the truncation radius. Note also that Romanova et al. (2002) have included viscosity in their simulations while we did not, with no 


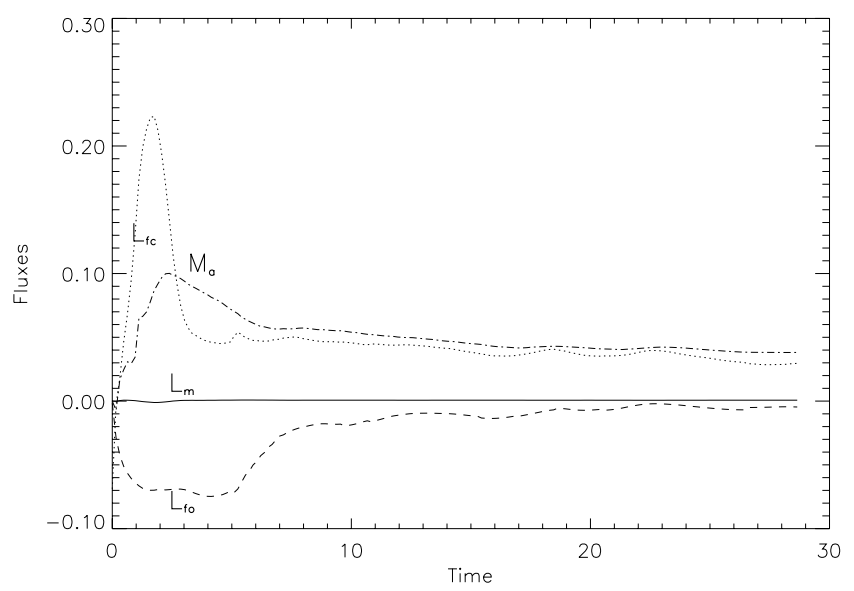

Fig. 5. Evolution in time of the accretion rate $\dot{M}_{a}$ (dash dotted line), angular momentum flux transported by matter $\dot{L}_{\mathrm{m}}$ (solid line) and by the magnetic field, computed for the closed $\dot{L}_{\mathrm{fc}}$ (dash line) and open $\dot{L}_{\mathrm{fo}}$ (dotted lines) field lines. The units are normalised and the two hemispheres are taken into account.

significant difference in the location of the truncation radius. To be more specific, using Eq. (6) with the higher accretion rate of $\dot{M}_{a}=9 \times 10^{-8} M_{\odot} y r^{-1}$ as measured by Romanova et al. (2002), we find $r_{\mathrm{t}, \text { th }}=0.9$ for $B_{*}=1.1 \mathrm{kG}$ using $m_{\mathrm{s}}=0.45$, which is consistent with the truncation radius shown Fig. 16 in Romanova et al. (2002) with a good accuracy. Furthermore, using Eq. (6) with the values provided by Kuker et al. (2003) one gets truncation radii smaller than the inner radial boundary, which explains why these authors did not find accretion columns. We are therefore confident on our main conclusion, that is the validity of our criterion (6).

We now consider the fluxes of angular momentum, namely $\dot{L}_{\mathrm{m}}$ carried in by the infalling material and $\dot{L}_{\mathrm{f}}$ by the magnetic field

$$
\begin{aligned}
& \dot{L}_{\mathrm{m}}=\int_{S} \rho \Omega r^{2} \boldsymbol{v}_{\mathrm{p}} \cdot \mathrm{d} \boldsymbol{S}=-4 \pi R_{*}^{2} \int_{S} \rho \Omega r^{2} v_{R} \sin \theta \mathrm{d} \theta \\
& \dot{L}_{\mathrm{f}}=-\int_{S} r B_{\phi} \boldsymbol{B}_{\mathrm{p}} \cdot \mathrm{d} \boldsymbol{S}=4 \pi R_{*}^{2} \int_{S} r B_{\phi} B_{R} \sin \theta \mathrm{d} \theta .
\end{aligned}
$$

To explicitly write these expressions we assumed that the surface element is directed inwards with respect to the surface of the star. The flux carried by the magnetic field is the sum of that carried by closed field lines and open field lines, namely $\dot{L}_{\mathrm{f}}=\dot{L}_{\mathrm{fc}}+\dot{L}_{\mathrm{fo}}$. It actually corresponds to two possible electric circuits each related to two different electromotive forces. A positive flux describes a positive torque acting on the star and leading to a spin up while a negative flux leads to a spin down. The time evolution of these fluxes is shown in Fig. 5 for our reference run (s1). Not surprisingly, the incoming angular momentum flux $\dot{L}_{\mathrm{m}}$ due to the accreting material is positive but totally negligible with respect to that carried by the closed magnetic field lines $\dot{L}_{\mathrm{fc}}$, which is positive as well: the accreting star is only being spun up. When looking more closely at $\dot{L}_{\mathrm{f}}$, it turns out that there is a negative magnetic contribution to the torque due to the open field lines $\dot{L}_{\mathrm{fo}}$. This has also been previously reported in simulations and is a natural outcome of the star-disc interaction (Long et al. 2005). We stress however that the actual torque is not controlled and one should not take it at face value. Indeed, this region of the magnetosphere should be the locus of a stellar wind but the physics of its launching has not yet been addressed. Finally, let us turn back to our result that the accretion rate $\dot{M}_{a}$ measured onto the star is about 10 times smaller than expected. In fact, $B_{*}$ and $\dot{M}_{a}$

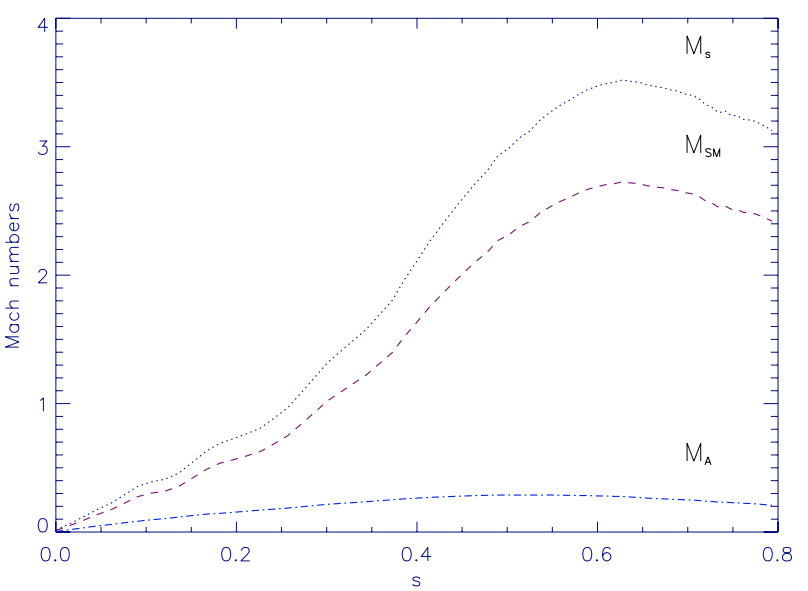

Fig. 6. Sonic (dotted line), slow-magnetosonic (dashed line) and Alfvénic (dash-dotted line) Mach numbers within the funnel flow along the same magnetic field line at $t=10$ as in Fig. 2.

were considered in Eq. (6) as independent parameters. However, the mass inflow at the inner edge of the accretion disc is constrained by the magnetic topology. Basically, the magnetosphere acts as a nozzle and the mass flow cannot be arbitrary. In a strict steady-state analysis it would be imposed by the regularity condition at the slow magnetosonic point. In our simulations, the flow reaches the slow point $M_{\mathrm{SM}}=1$ above the disc at $s \sim 0.3$ (see Fig. 6). Then, it remains supersonic with a maximum for the sonic Mach number around $M_{s} \sim 3.5$ but the funnel flow is always sub-Alfvénic with a maximum Alfvénic Mach number $M_{\mathrm{A}} \sim 0.3$ near the middle path between the stellar surface and the disc inner edge.

\section{Conclusion}

We have confirmed that the formation of accretion funnel flows is a robust feature of axisymmetric star-disc interactions. We investigated the physical conditions required to produce steadystate funnel flows onto a dipole and provided an analytic expression of the disc truncation radius. We then used MHD simulations with VAC to show its validity.

Our theoretical expression $r_{t, t h}$ relates the disc truncation radius to astrophysical parameters such as stellar dipole field $B_{*}$, mass $M_{*}$, radius $R_{*}$ and accretion rate $\dot{M}_{a}$. Although it resembles the Alfvén radius (Elsner \& Lamb 1977) sometimes invoked in similar situations (Bouvier et al. 2007), it has been physically motivated on very different ground. It is shown that it gives an accurate prediction of the real truncation radius as obtained in current 2D MHD simulations of a star-disc interaction.

We report MHD simulations displaying accretion funnels with a weak stellar dipole field $B_{*} \sim 140 \mathrm{G}$ from a resistive non-viscous disc. In this case, the disc inner edge is found closer to the star, below the co-rotation radius, in agreement with the size of inner disc holes. However, the accretion rate onto the star which is imposed by the physics of magnetic accretion is measured to be $\dot{M} \sim 10^{-9} M_{\odot} \mathrm{yr}^{-1}$. Even though this magnitude of accretion rate is found for some T Tauri stars (see Gullbring et al. 1998), this result shows that it is necessary to have stronger fields (by a factor of around 7) to have magnetospheric accretion for typical mean accretion rates of $\dot{M} \sim 10^{-8} M_{\odot} \mathrm{yr}^{-1}$. On the other hand, the few circular polarization measurements available, provide an upper limit of 100-200 G for the dipolar component. However, recent spectro-polarimetric observations coupled with magnetic field reconstruction conducted on 2 CTTS V2129 Oph 
and BP Tau provide higher dipole field components of $350 \mathrm{G}$ and $1.2 \mathrm{kG}$ respectively (Donati et al. 2007a, b in prep.). In any case, if the presence of $\mathrm{kG}$ dipole fields around CTTS are indeed ruled out, the requirement of forming quasi-steady accretion funnels imposes that the magnetic topology must be different. This is a firm result based on our dynamical calculations. One then needs to consider other stellar magnetic field topologies such as multipolar components and/or an inclined dipole configuration (Romanova et al. 2003; Long et al. 2007). In this paper, since there is no viscosity on our resistive accretion disc, we privileged a situation where $r_{\mathrm{t}} \ll r_{\mathrm{co}}$ to reach an open magnetic topology within the main part of the disc. This made it possible to have a braking torque feeding the disc even without viscosity. Apart from the localised magnetic flux channeling the accretion funnel, the other stellar magnetic field lines become causally disconnected from the disc. This forbids any disc-locking mechanism and the star is being spun up as a consequence of accretion. We also report the non development of X-winds despite the favourable topology. Both of these aspects deserve however a more detailed analysis as they may depend on the disc resistivity. While the disc truncation radius remained close to its predicted position for about 25 Keplerian periods at the disc inner edge, some variability is obviously taking place. This is a very promising topic as veiling measurements probing accretion onto the star do show variability on different time scales (Alencar \& Basri 2000). However making longer simulations requires one to implement the turbulent viscosity to allow for accretion within the disc beyond corotation. This work is in progress.

Acknowledgements. We acknowledge useful suggestions and constructive criticism of an anonymous referee on a first version of this paper. We thank the computational facilities of the Service Commun de Calcul Intensif de l'Observatoire de Grenoble (SCCI). The present work was supported in part by the European Community Marie Curie Actions - Human Resource and Mobility within the JETSET (Jet Simulations, Experiments and Theory) network under contract MRTN-CT-2004 005592.

\section{References}

Alencar, S. H. P., \& Basri, G. 2000, AJ, 119, 119, 1881

Aly, J. J. 1980, A\&A, 86, 192

Aly, J. J., \& Kuijpers, J. 1990, A\&A, 227, 473

Armitage, P. J., \& Clarke, C. J. 1996, MNRAS, 280, 458
Arons, J. 1993, ApJ, 408, 160

Blandford, R. D., \& Payne, D. G. 1982, MNRAS, 199, 883

Bouvier, J., Chelli, A., Allain, S., et al. 1999, A\&A, 349, 619

Bouvier, J., Grankin, K. N., Alencar, S. H. P., et al. 2003, A\&A, 409, 169

Bouvier, J., Alencar, S. H. P., Harries, T. J., Johns-Krull, C. M., \& Romanova,

M. M. 2007, in Protostars and Planets V, ed. B. Reipurth, D. Jewitt, \& K.

Keil, 479

Casse, F., \& Keppens, R. 2002, ApJ, 581, 988

Cameron, A. C., \& Campbell, C. G. 1993, A\&A, 274, 309

Donati, J.-F., Jardine, M. M., Gregory, S. G., et al. 2007, MNRAS, 380, 1297

Edwards, S., Hartigan, P., Ghandour, L., \& Andrulis, C. 1994, AJ, 108, 1056

Elsner, R. F., \& Lamb, F. K. 1977, ApJ, 215, 897

Ferreira, J., \& Pelletier, G. 1995, A\&A, 295, 807

Ghosh, P., \& Lamb, F. K. 1979, ApJ, 232, 259

Gregory, S. G., Jardine, M., Simpson, I., \& Donati, J.-F. 2006, MNRAS, 371, 999

Gullbring, E., Hartmann, L., Briceno, C., \& Calvet, N. 1998, ApJ, 492, 323

Hayashi, M. R., Shibata, K., \& Matsumoto, R. 1996, ApJ, 468, L37

Johns-Krull, C. M., Valenti, J. A., Hatzes, A. P., \& Kanaan, A. 1999, ApJ, 510, L41

Königl, A. 1991, ApJ, 370, L39

Küker, M., Henning, T., \& Rüdiger, G. 2003, ApJ, 589 , 397

Koldoba, A. V., Lovelace, R. V. E., Ustyugova, G. V., \& Romanova, M. M. 2002, AJ, 123, 2019

Long, M., Romanova, M. M., \& Lovelace, R. V. E. 2005, ApJ, 634, 1214

Long, M., Romanova, M. M., \& Lovelace, R. V. E. 2007, MNRAS, 374, 436

Lovelace, R. V. E., Romanova, M. M., \& Bisnovatyi-Kogan, G. S. 1995, MNRAS, 275, 244

Matt, S., \& Pudritz, R. E. 2005, MNRAS, 356, 167

Mignone, A., Bodo, G., Massaglia, S., et al. 2007, ApJS, 170, 228

Miller, K. A., \& Stone, J. M., 1997, ApJ, 489, 890

Najita, J., Carr, J. S., \& Mathieu, R. D. 2003, ApJ, 589, 931

Ostriker, E. C., \& Shu, F. H. 1995, ApJ, 447, 813

Powell, K. G., Roe, P. L., Linde, T. J., Gombosi, T. I., \& de Zeeuw, D. L. 1999, J. Comp. Phys., 154, 284

Pringle, J. E., \& Rees, M. J. 1972, A\&A, 21, 1

Romanova, M. M., Ustyugova, G. V., Koldoba, A. V., \& Lovelace, R. V. E. 2002, ApJ, 578, 420

Romanova, M. M., Ustyugova, G. V., Koldoba, A. V., Wick, J. V., \& Lovelace, R. V. E. 2003, ApJ, 595, 1009

Shakura, N. I., \& Syunyaev, R. A. 1973, A\&A, 24, 337

Shu, F., Najita, J., Ostriker, E., Wilkin, F., Ruden, S., \& Lizano, S. 1994, ApJ, 429, 781

Tanaka, T. 1994, J. Comp. Phys., 111, 381

Tóth, G. 1996, Astrophys. Lett. Comm., 34, 245

Ustyugova, G. V., Koldoba, A. V., Romanova, M. M., \& Lovelace, R. V. E. 2006, ApJ, 646, 304

Valenti, J. A. \& Johns-Krull, C. M. , 2004, Ap\&SS, 292, 619

Wang, Y. M. 1996, ApJ, 465, 111

Zanni, C., Ferrari, A., Rosner, R., Bodo, G., \& Massaglia, S. 2007, A\&A, 469, 811 